\title{
Pediatric Cancer Variant Pathogenicity Information Exchange (PeCanPIE): a cloud-based platform for curating and classifying germline variants
}

\author{
Michael N. Edmonson, ${ }^{1,11}$ Aman N. Patel, ${ }^{1,11}$ Dale J. Hedges, ${ }^{1}$ Zhaoming Wang, ${ }^{1}$ \\ Evadnie Rampersaud, ${ }^{1}$ Chimene A. Kesserwan, ${ }^{2}$ Xin Zhou, ${ }^{1}$ Yanling Liu, ${ }^{1}$ \\ Scott Newman, ${ }^{1}$ Michael C. Rusch, ${ }^{1}$ Clay L. McLeod, ${ }^{1}$ Mark R. Wilkinson, ${ }^{1}$ Stephen \\ V. Rice, ${ }^{1}$ Thierry Soussi, ${ }^{3,4,5}$ J. Paul Taylor, ${ }^{6,7}$ Michael Benatar, ${ }^{8}$ Jared B. Becksfort, ${ }^{1}$ \\ Kim E. Nichols, ${ }^{2}$ Leslie L. Robison, ${ }^{9}$ James R. Downing, ${ }^{10}$ and Jinghui Zhang ${ }^{1}$ \\ ${ }^{1}$ Department of Computational Biology, ${ }^{2}$ Department of Oncology, St. Jude Children's Research Hospital, Memphis, Tennessee \\ 38105, USA; ${ }^{3}$ Sorbonne Université, UPMC Univ Paris 06, F-75005 Paris, France; ${ }^{4}$ Department of Oncology-Pathology, Cancer Center \\ Karolinska (CCK), Karolinska Institutet, 17164 Stockholm, Sweden; ${ }^{5}$ INSERM, U1138, Équipe 11, Centre de Recherche des \\ Cordeliers, 75006 Paris, France; ${ }^{6}$ Howard Hughes Medical Institute, Chevy Chase, Maryland 20815, USA; ${ }^{7}$ Department of Cell and \\ Molecular Biology, St. Jude Children's Research Hospital, Memphis, Tennessee 38105, USA; ${ }^{8}$ Department of Neurology, University of \\ Miami, Miami, Florida 33136, USA; ${ }^{9}$ Department of Epidemiology and Cancer Control, ${ }^{10}$ Department of Pathology, St. Jude \\ Children's Research Hospital, Memphis, Tennessee 38105, USA
}

\begin{abstract}
Variant interpretation in the era of massively parallel sequencing is challenging. Although many resources and guidelines are available to assist with this task, few integrated end-to-end tools exist. Here, we present the Pediatric Cancer Variant Pathogenicity Information Exchange (PeCanPIE), a web- and cloud-based platform for annotation, identification, and classification of variations in known or putative disease genes. Starting from a set of variants in variant call format (VCF), variants are annotated, ranked by putative pathogenicity, and presented for formal classification using a decision-support interface based on published guidelines from the American College of Medical Genetics and Genomics (ACMG). The system can accept files containing millions of variants and handle single-nucleotide variants (SNVs), simple insertions / deletions (indels), multiple-nucleotide variants (MNVs), and complex substitutions. PeCanPIE has been applied to classify variant pathogenicity in cancer predisposition genes in two large-scale investigations involving $>4000$ pediatric cancer patients and serves as a repository for the expert-reviewed results. PeCanPIE was originally developed for pediatric cancer but can be easily extended for use for nonpediatric cancers and noncancer genetic diseases. Although PeCanPIE's web-based interface was designed to be accessible to non-bioinformaticians, its back-end pipelines may also be run independently on the cloud, facilitating direct integration and broader adoption. PeCanPIE is publicly available and free for research use.
\end{abstract}

[Supplemental material is available for this article.]

Massively parallel sequencing has quickly become a mainstay for genetic variation studies in many research and clinical genomics laboratories. However, the sheer abundance of data produced for a single individual means that complex and often tedious data processing and curation are required to identify potentially diseasecausing mutations. The process is simultaneously burdened by the volume of novel variants, many of which have scarce information available, and the diverse, distributed nature of existing variant information resources. Variant annotation tools have been developed to assist with several aspects of this work, which can add coding and noncoding predictions and population-specific allele frequencies, as well as provide filtering options for variant prioritization (Ng et al. 2009; Wang et al. 2010; Cingolani et al. 2012; McLaren et al. 2016). Likewise, variant curation tools supporting

\footnotetext{
${ }^{11}$ These authors contributed equally to this work. Corresponding author: Jinghui.Zhang@stjude.org Article published online before print. Article, supplemental material, and publication date are at http://www.genome.org/cgi/doi/10.1101/gr.250357.119. Freely available online through the Genome Research Open Access option.
}

classification for clinical pathogenicity following the American College of Medical Genetics and Genomics (ACMG) guidelines (Richards et al. 2015) have also been developed (Patel et al. 2017). Although each resource offers valuable information to help researchers classify variant pathogenicity, integrated platforms are needed to provide support for all steps of the process and to streamline analysis of the thousands to millions of variants generated by massively parallel sequencing technology. With these goals in mind, we developed the Pediatric Cancer Variant Pathogenicity Information Exchange (PeCanPIE), a cloud-based portal that provides an end-to-end workflow, beginning with a set of variants in VCF (Danecek et al. 2011) and ending with ACMG-compliant classification. PeCanPIE offers three key functions: (1) automated annotation, classification, and triage via our MedalCeremony pipeline (Zhang et al. 2015); (2) an interactive variant page and visualization tools to support expert curation and committee review; and (3) a reference database of expert-reviewed germline cancer-

(C) 2019 Edmonson et al. This article, published in Genome Research, is available under a Creative Commons License (Attribution 4.0 International), as described at http://creativecommons.org/licenses/by/4.0/. 
predisposing mutations. PeCanPIE is designed on the theme of genetic variant analysis, making it flexible for extending its application to noncancer-related genetic diseases.

\section{Results}

\section{Process overview}

As outlined in Figure 1A, PeCanPIE launches with an interface for uploading a VCF file; alternatively, a single variant may be specified either genomically or in HGVS RNA format, which is translated into genomic coordinates via Mutalyzer (Wildeman et al. 2008). The system is native to GRCh37; GRCh38 variants are also accepted and mapped to their GRCh37 equivalent via the UCSC liftOver utility (Hinrichs et al. 2006). PeCanPIE makes use of predicted protein-coding impacts, which are expected to be identical for both reference genomes. Uploaded variants are then filtered to a set of disease-related genes (Methods; Supplemental Table S1). Users may select from predefined lists of genes from several disease categories, including cancer, cardiovascular, nonmalignant hematological, immunodeficiency, and amyotrophic lateral sclerosis (ALS) and related disorders. Alternatively, users may specify their own candidate gene list for analysis. Variants are next assigned gene and protein annotations with the Ensembl Variant Effect Predictor (VEP) pipeline (McLaren et al. 2016) and filtered by functional class and population frequency derived from the Exome
Aggregation Consortium (ExAC) database (Lek et al. 2016). To ensure that pathogenic germline variants in cancer patients are retained, PeCanPIE uses the non-TCGA subset of ExAC that excludes patient samples from The Cancer Genome Atlas (TCGA) (The Cancer Genome Atlas Research Network 2008). The remaining variants are stratified into three tiers (gold, silver, and bronze) as an indication of potential pathogenicity computed by our MedalCeremony pipeline (see below). Finally, each "medaled" variant is linked to a stand-alone page featuring an interface to support semiautomated pathogenicity classification using ACMG guidelines. Two examples in Figure 1 demonstrate the classification process using VCF files generated from whole-exome sequencing (WES) of an acute lymphoblastic leukemia (ALL) patient (Fig. 1B; Moriyama et al. 2015) and whole-genome sequencing (WGS) from the Genome in a Bottle (GIAB) project (Fig. 1C; Zook et al. 2014), respectively. Only 14 of the 63,109 variants from the WES data and 17 of the approximately 4 million variants from the WGS data required expert review, which resulted in 1 and 0 pathogenic/likely pathogenic (P/LP) variants, respectively.

\section{Automated classification by the MedalCeremony pipeline}

Automated classification of variant pathogenicity implemented in the MedalCeremony pipeline classifies variants that have a population frequency of $\leq 0.001$ in the ExAC non-TCGA database. If desired, the frequency cutoff can be adjusted by the user or disabled

\section{PIE Process Overview}

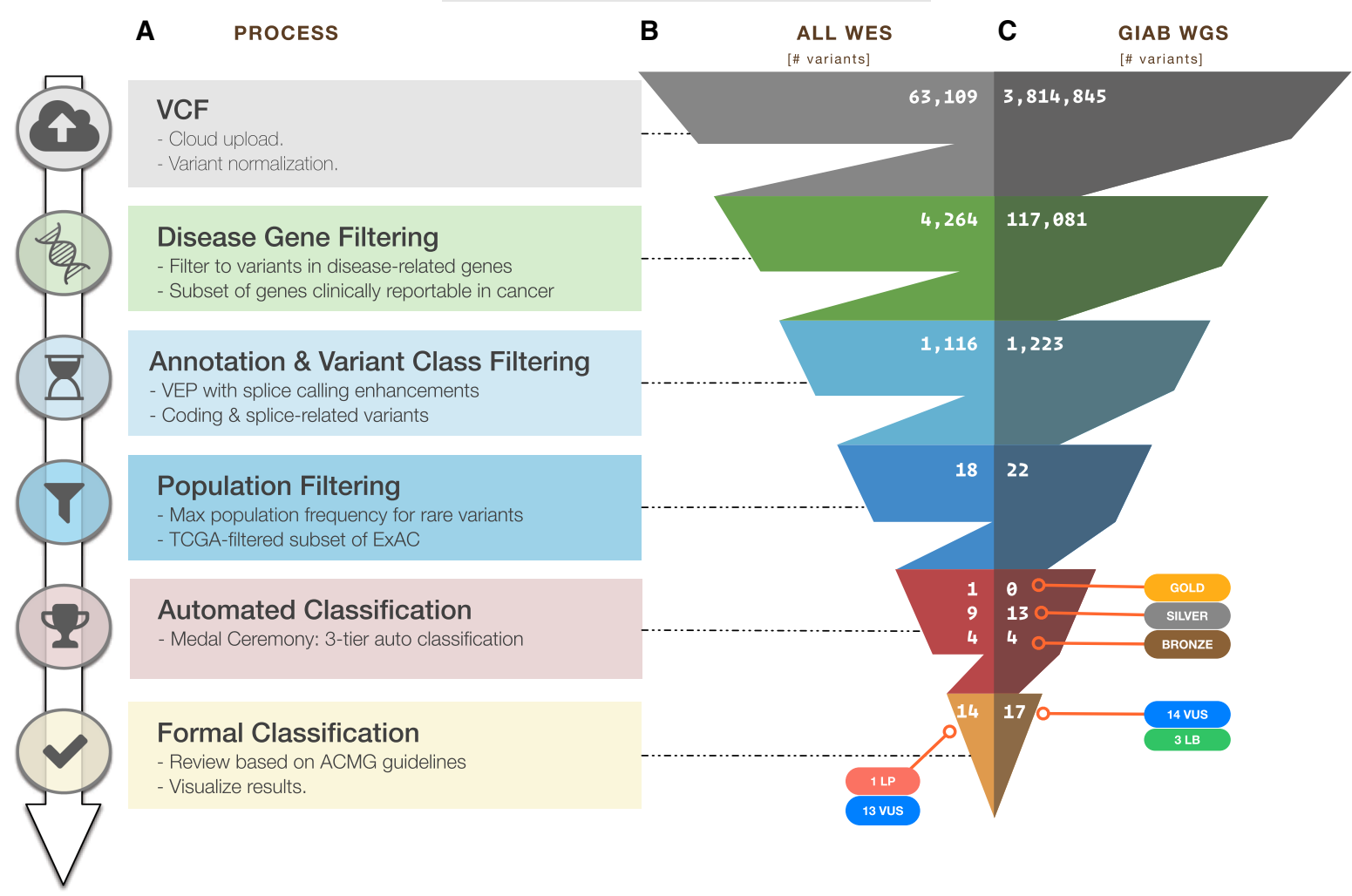

Figure 1. Overview of variant classification using PeCanPIE. (A) Overview of processing steps from VCF through ACMG-based classification. Variant counts at each processing step for whole-exome sequencing data generated from a germline sample of a patient with acute lymphoblastic leukemia (ALL), SJNORM015857_G1 (Methods) (B) and whole-genome sequencing data generated from Genome in a Bottle normal sample NA12878_HG001 (Methods) (C).

\section{Genome Research}

www.genome.org 
altogether. Rather than the standard distribution, the non-TCGA subset of ExAC is used because as a large collection of cancer samples, TCGA is likely to be enriched for mutations related to cancer predisposition; for example, germline pathogenic mutations were previously detected in TCGA ovarian cancer samples for BRCA1, $B R C A 2$, and PALB2 (Kanchi et al. 2014). Variants appearing at a frequency higher than the cutoff may still be classified if they are present either in the International Agency for Research on Cancer (IARC) TP53 database or in ClinVar with a classification of pathogenic or likely pathogenic and a review status of two or more gold stars (https://www.ncbi.nlm.nih.gov/clinvar/docs/review_status/). The latter exception helps retain pathogenic variants that may appear at higher population frequencies owing to founder effects, partial penetrance, or other factors impacting phenotypic heterogeneity and age at onset. To support incorporation of custom data for variant classification, users may optionally provide a "whitelist" of custom variants, matches to which will always receive a medal, or a "blacklist," matches to which will be prevented from receiving a medal. Additional annotations are incorporated to aid with the classification process: (1) COSMIC (Forbes et al. 2008) hits; (2) functional annotations from dbNSFP (protein domain and damage-prediction algorithm calls) (Liu et al. 2013); and (3) allele frequencies in the NHLBI GO Exome Sequencing Project (ESP), the 1000 Genomes Project (The 1000 Genomes Project Consortium 2015), ExAC non-TCGA, and the Pediatric Cancer Genome Project (PCGP) (Downing et al. 2012). Although COSMIC and PCGP represent somatic rather than germline data sets, these can help inform germline classification; an example is discussed below in the "ACMG classification interface" section.

An overview of the gold, silver, and bronze classification scheme implemented in MedalCeremony is shown in Figure 2. Gold medals are assigned to truncating variants (including splice variants) in genes where loss-of-function variants are associated with a disease (e.g., tumor suppressor genes in cancer) (Zhao et al. 2016; Chakravarty et al. 2017) as well as matches to highly curated databases, including IARC TP53 (Bouaoun et al. 2016), ClinVar pathogenic (P) or likely pathogenic (LP) variants with a review status of two or more gold stars, Arizona State University (ASU) TERT (Podlevsky et al. 2007), the University of Utah Department of Pathology (ARUP) MEN2 (Margraf et al. 2009), and the National Human Genome Research Institute (NHGRI) Breast Cancer Information Core (Szabo et al. 2000). Gold medals are also assigned to somatic mutation hotspots in COSMIC (observed in $\geq 10$ tumors after removal of hypermutators) and PCGP, St. Jude committee-reviewed germline P/LP variants, and user-provided whitelist variants, if specified. Silver medals are assigned to in-frame indels, truncation events in non-tumor suppressor genes, variants predicted to be damaging by in silico algorithms, and matches to additional databases: ClinVar P/LP with fewer than two gold stars, BRCA
Share (Béroud et al. 2016), ALSoD (Abel et al. 2013), Leiden Open Variation Database (LOVD) (Fokkema et al. 2011) locus-specific databases for APC and MSH2, and RB1 (Lohmann and Gallie 1993). Unless otherwise medaled, variants predicted to be tolerated by in silico algorithms are assigned a bronze medal. Imperfect database matches (e.g., a different allele at the same genomic position or at the same codon but with a different amino acid change) are typically assigned a lower-grade medal, for example, silver rather than gold. Variants not meeting any of the previous criteria, for example, most silent variants and those without any functional annotations, will not receive a medal. Amino acid and pathogenicity codes from the diverse variant databases used in this process are standardized to improve the reliability of annotations and utility of information (Methods). A summary of resources is shown in Table 1. MedalCeremony may also be run as a stand-alone pipeline on St. Jude Cloud (Methods).

\section{Variant review interface}

After MedalCeremony classification, the results are presented in a table that can be searched or filtered by gene, variant class, medal, or expert review classification (Fig. 3A). If a variant has been previously classified by the user or the St. Jude germline variant review committee, that information will be prepopulated. Variant classifications previously performed by each user are also prepopulated, allowing groups working on other diseases to establish their own expert review committees (see "Variant classification of noncancer genes" below). Each row links to a variant page containing extensive annotations, including gene information from the National

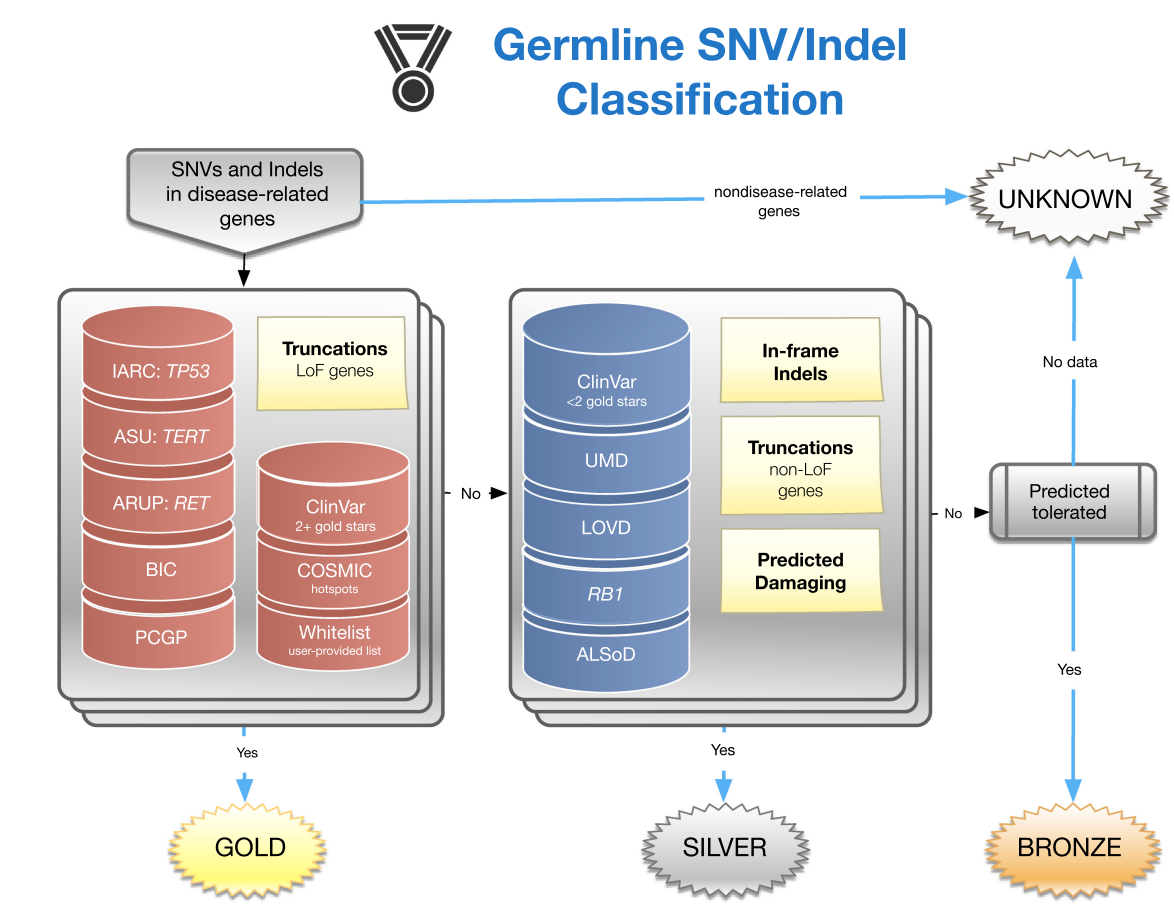

Figure 2. Design of the MedalCeremony pipeline for automated germline variant classification. Truncating variants in loss-of-function genes (e.g., tumor suppressors) and those matching highly curated databases receive gold medals. Truncations in non-loss-of-function genes, in-frame indels, variants predicted to be damaging, and matches to additional databases receive silver medals. Otherwise, variants predicted to be tolerated by damage-prediction algorithms receive bronze medals. Imperfect database matches receive a lower-grade medal than exact matches. Variants not meeting any of the prior criteria are labeled "unknown." 
Table 1. Databases used in classification

\begin{tabular}{|c|c|}
\hline Source & URL \\
\hline ClinVar & https://www.ncbi.nlm.nih.gov/clinvar/ \\
\hline dbNSFP & https://sites.google.com/site/jpopgen/dbNSFP \\
\hline ExAC non-TCGA & $\begin{array}{l}\text { ftp://ftp.broadinstitute.org/pub/ExAC_release/ } \\
\text { release1/subsets/ }\end{array}$ \\
\hline COSMIC & https://cancer.sanger.ac.uk/cosmic/ \\
\hline IARC TP53 & http://tp53.iarc.fr/ \\
\hline St. Jude PCGP & https://pecan.stjude.cloud/pcgp-explore \\
\hline ALSoD & http://alsod.iop.kcl.ac.uk/ \\
\hline NHGRI BIC & https://research.nhgri.nih.gov/bic/ \\
\hline LOVD RB1 & http://rb1-lovd.d-lohmann.de/ \\
\hline BRCA Share & http://www.umd.be/BRCA1/ \\
\hline ASU TERT & http://telomerase.asu.edu/diseases.html\#tert \\
\hline $\begin{array}{l}\text { University of Utah } \\
\text { MEN2 }\end{array}$ & $\begin{array}{l}\text { http://www.arup.utah.edu/database/MEN2/ } \\
\text { MEN2_display.php }\end{array}$ \\
\hline LOVD $A P C, M S H 2$ & https://databases.lovd.nl/shared/diseases/05489 \\
\hline
\end{tabular}

Center for Biotechnology Information (NCBI) and the Online Mendelian Inheritance in Man (OMIM) database (Amberger et al. 2015), ClinVar match details, and population frequencies (Fig. 3B). Cancer predisposition genes are classified as autosomal dominant and autosomal recessive (Zhang et al. 2015) and annotated as such on the interface; this is used for determining the status of a patient but not during automated classification of individual variants. For example, in autosomal recessive genes, compound heterozygosity will be considered as having the same effect as homozygosity when a patient is signed out; this is determined when reviewing all data collected from the patient. Via dbNSFP, in silico predictions of deleteriousness are included (Fig. 3C), including REVEL (Ioannidis et al. 2016) pathogenicity scores, which fared well in a comparison of algorithms for use with ACMG clinical variant interpretation guidelines (Ghosh et al. 2017). The page also includes an embedded ProteinPaint view (Zhou et al. 2015), which overlays the current variant and other user-uploaded variants in the same data set with aggregated somatic mutations and expert-classified P/LP germline variants on the protein product. This enables visual inspection of variant recurrence, hotspots, and enrichment of loss-of-function mutations. For TP53, data from several variant-level databases of functional activity (Soussi et al. 2006; Giacomelli et al. 2018; Kotler et al. 2018) are presented to aid reviewers in assessing pathogenicity. Examples of pathogenic and benign variants are shown in Figure 3D; additional examples, including those classified as variant of uncertain significance (VUS) by our analysis (e.g., TP53 P222L), can be explored on our portal (https://pecan.stjude.cloud/variant/67664). In addition to TP53, we have also incorporated published functional data from IKZF1 (Churchman et al. 2018) and will continue to expand the repertoire of functional data generated by basic research scientists.

\section{ACMG classification interface}

A powerful feature of the variant detail page is an interactive graphical interface that allows a reviewer to enter a series of pathogenicity criteria evidence tags (e.g., population frequency, segregation, functional significance, and in silico prediction), along with supporting information such as PubMed IDs, to automatically calculate a five-tier classification: pathogenic (P), likely pathogenic (LP), variant of unknown significance (VUS), likely benign (LB), and benign (B) based on the ACMG algorithm. MedalCeremony can automatically generate ACMG classification tags for variants, which are prepopulated into PeCanPIE's classification interface.
The following automatic tags are implemented per their ACMG specifications: PVS1 (truncating variant in a tumor suppressor or other loss-of-function gene), PM1 (somatic hotspot in COSMIC), PM2 (absent from ExAC non-TCGA or appearing at a frequency not greater than 0.0001) and the companion BA1 tag ( $>5 \%$ population frequency in ExAC non-TCGA), PM4 (in-frame protein insertions and deletions), PS1, and PM5 (amino acid comparisons made vs. pathogenic variants in ClinVar or those identified by the St. Jude germline variant review committee). Automatically assigned tags may be removed by the analyst if desired. This automation provides improved support over manual curation interfaces while still retaining analyst control over the ultimate classification decisions. As shown on the variant page for ETV6 Arg359Ter, the single gold medal variant detected in the patient with ALL was expert-classified as likely pathogenic because the mutation is present in a disease-related gene (i.e., ETV6 is a pediatric ALL driver gene), is a loss-of-function null variant, and is not present in the ExAC non-TCGA database (Fig. 4).

Comparison of a germline variant with aggregated somatic variants can help inform germline classification for cancer predisposition genes. For example, family studies have identified a PAX5 G183S germline mutation conferring susceptibility to B-ALL, which corresponds to somatic mutations detected in pediatric BALL and lymphoma (Shah et al. 2013). A similar profile was observed in the example WES data from an ALL patient presented in Figure 1B: MedalCeremony assigned a single gold medal—a novel ETV6 nonsense variant within the ETS domain (NM_001987.4: c.1075C > T, NP_001978.1:p.Arg359Ter)—based on the criteria of truncation in a tumor suppressor gene. The ProteinPaint view embedded in the variant page confirmed that in ETV6, somatic mutations are dominated by loss-of-function mutations across pediatric leukemia (Fig. 4), consistent with the tumor suppressor gene model. This pathogenic variant was discovered in a research project, in which the MedalCeremony pipeline flagged this variant repeatedly in an affected family. Reviewers may enter custom evidence such as this into the interface for use during final classification.

Recurrent mutations, which include many gain-of-function variants, are reported either through matches to somatic mutation hotspots or to pathogenic variants in curated germline databases, such as ClinVar. Figure 5 shows a germline NRAS G12S variant detected in one patient to illustrate this case. Somatic data rendered in the ProteinPaint view shows a hotspot at G12, and automated classification detects pathogenic variations at the same amino acid position in ClinVar, as well as a hotspot in COSMIC data. This, coupled with the fact that biochemical assays have shown that this mutation would activate RAS (Schubbert et al. 2007), the final classification by the committee is pathogenic.

Collaborative features are also available: Users may invite others to access their results and work together on classification. This helps multidisciplinary researchers in disparate locales to perform distributed review and form their own variant classification committees if desired.

\section{Variant classification of noncancer genes}

Using the collaborative features described above, we are in the process of classifying and reviewing variants with the Clinical Research in ALS and Related Disorders for Therapeutic Development (CReATe) Consortium. To illustrate the utility of PeCanPIE to disease areas outside the realm of cancer genomics, we show the classification of a variant in the superoxide dismutase 1 (SOD1) gene reported by the ALS research community in the ALSoD

\section{Genome Research}

www.genome.org 
A RESULTS PAGE - GIAB WGS

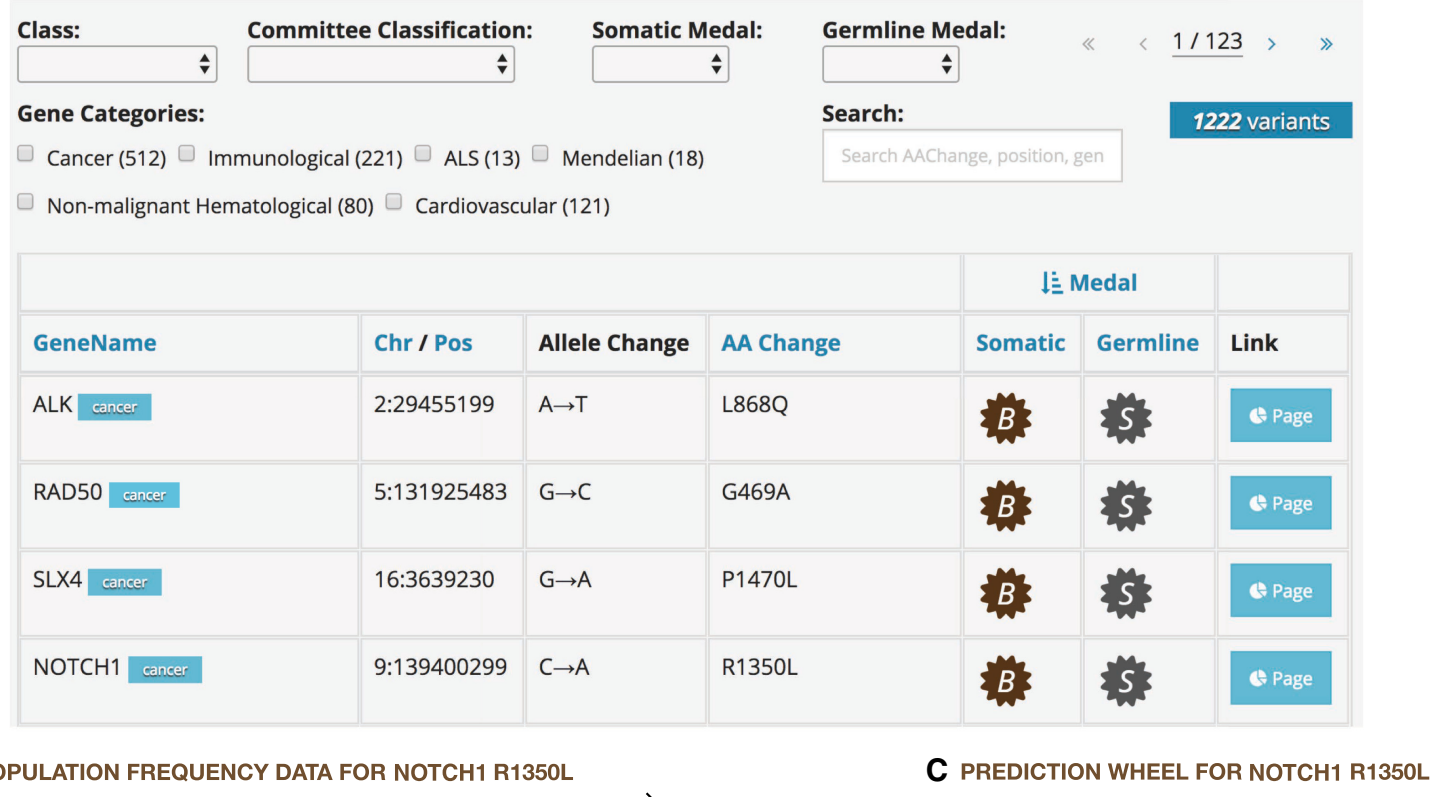

B POPULATION FREQUENCY DATA FOR NOTCH1 R1350L

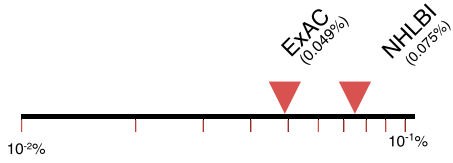

\begin{tabular}{lrrr}
\hline Popluation & Allele Frequency & Allele Count & \multicolumn{2}{r}{ Allele Number } \\
\hline Adjusted & $0.06216 \%$ & 49 & 78830 \\
\hline African/African American & $0 \%$ & 0 & 5344 \\
\hline East Asian & $0 \%$ & 0 & 6230 \\
\hline Finnish & $0.08772 \%$ & 3 & 3420 \\
\hline Latino & $0 \%$ & 0 & 8266 \\
\hline Non-Finnish European & $0.09522 \%$ & 40 & 42010 \\
\hline Other & $0 \%$ & 0 & 482 \\
\hline South Asian & $0.04588 \%$ & 6 & 13078 \\
\hline TOTALS & $\mathbf{0 . 0 4 9 0 4 \%}$ & $\mathbf{5 1}$ & $\mathbf{1 0 3 9 8 8}$ \\
\hline
\end{tabular}

PolyPhen2 HVAR CADD Benign $7.173($ raw $=0.47)$

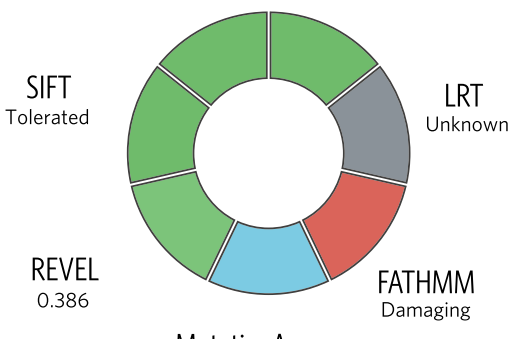

MutationAssessor

Low

D TP53 functional assays for N235S and G245S
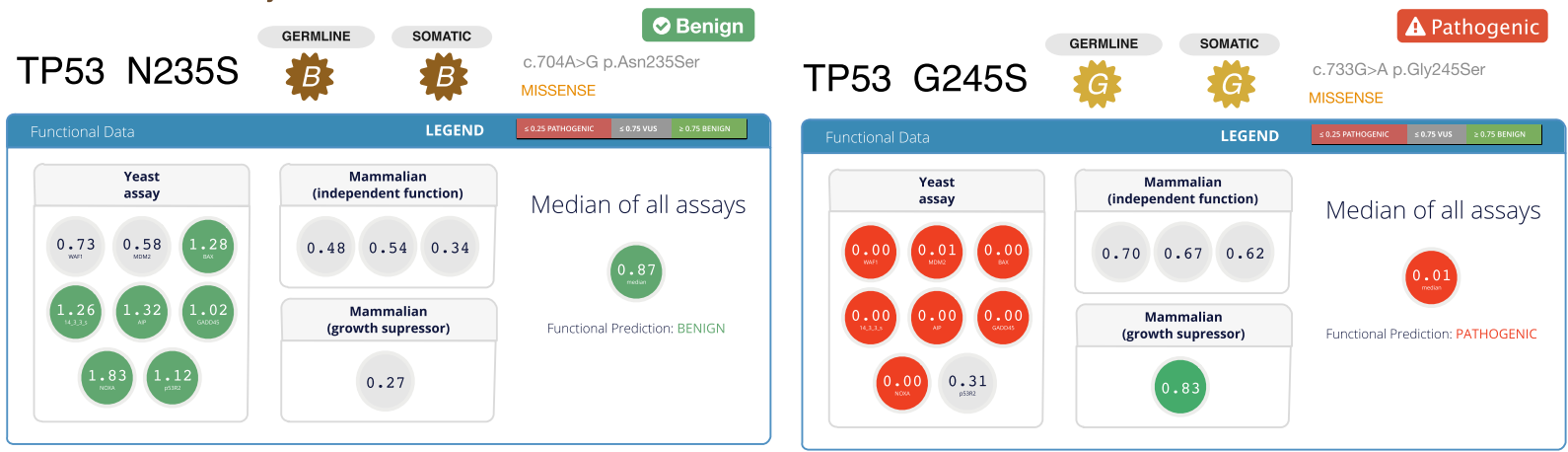

Figure 3. Annotation interface. Excerpts of PeCanPIE annotation interface. (A) Results for Genome in a Bottle WGS data set. Variant page details for NOTCH1 R1350L: $(B)$ variant population frequency detail from ExAC non-TCGA database; $(C)$ functional predictions. $(D)$ Functional data display for TP53 gene: Functional assay results for N235S and G245S show that N235S appears functionally benign, whereas G245S appears functionally damaging. 

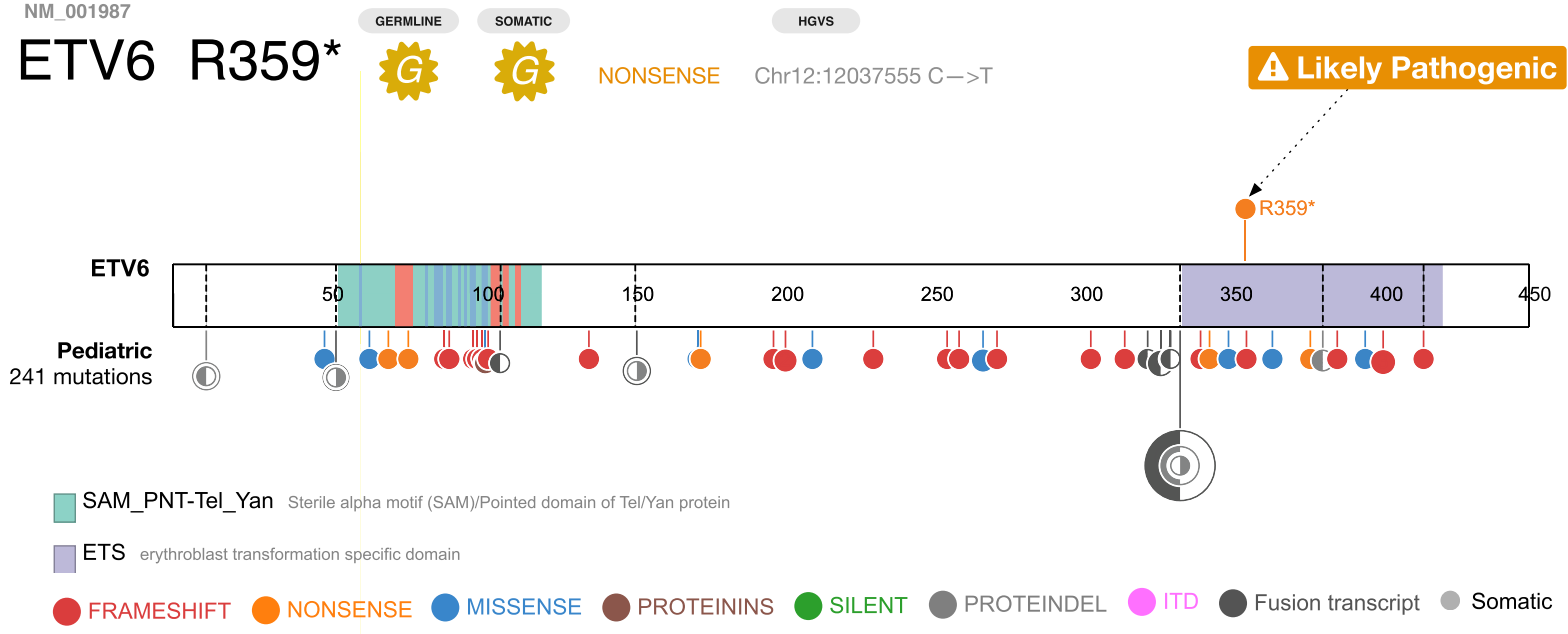

\begin{tabular}{ll}
\hline Category & Evidence \& Reference \\
\hline PVS1 - Very Strong 4 & $\begin{array}{l}\text { PVS1 null variant (nonsense, frameshift, canonical } \pm 1 \text { or 2 splice } \\
\text { sites, initiation codon, single or multiexon deletion) in a gene } \\
\text { where LOF is a known mechanism of disease. }\end{array}$ \\
\hline PM2 - Moderate 4 & $\begin{array}{l}\text { Absent from controls (or at extremely low frequency if } \\
\text { recessive) in Exome Sequencing Project, 1000 Genomes } \\
\text { Project, or Exome Aggregation Consortium }\end{array}$ \\
\hline
\end{tabular}

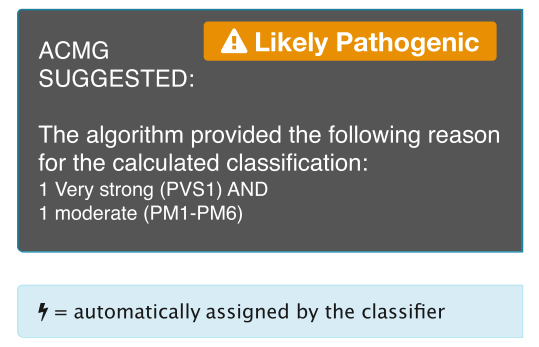

Figure 4. ACMG classification on ETV6. (Top) ProteinPaint display of somatic ETV6 variants across 11 subtypes of pediatric leukemia, showing enrichment of loss-of-function mutations (frameshifts in red, nonsense variants in orange). Arrow indicates position of germline R359* variant. (Bottom) Detail of PeCanPIE ACMG classification interface for R359* variant.

database. Following ACMG classification criteria and taking into account the disease model as reported in OMIM, researchers at St. Jude are performing initial classifications, which undergo subsequent review by external researchers via secure remote login. As an example, we show a gold standard variant Ala5Val in the superoxide dismutase 1 (SOD1) gene (Fig. 6), located on Chromosome 21, which is the most common ALS-causing mutation in the United States (Rosen et al. 1994; Cudkowicz et al. 1997; Valentine and Hart 2003). Of note, the variant is displayed with reference to all variants in SOD1 in the uploaded ALSoD data set, making it easy for researchers to determine the clustering of such variants for downstream targeted analyses. Using the semiautomated ACMG classification, we were able to determine that this variant is pathogenic as expected. Additional PubMed identifiers have been included in the ACMG PS3 criterion to allow external viewers to weigh the evidence accordingly.

\section{Use of PeCanPIE by the genetic research community}

PeCanPIE was initially designed in support of large-scale germline variation analysis projects and was iteratively improved based on the feedback of an interdisciplinary group of researchers and individual users. Germline variants from the following studies have been analyzed thus far: (1) A study of germline variations in predisposition genes in 1120 children with cancer (Zhang et al. 2015) classified 890 variants, identifying 109 as pathogenic (P) and 25 as likely pathogenic (LP); (2) the St. Jude LIFE project, a follow-up study of 3006 long-term survivors of pediatric cancer
(Wang et al. 2018), classified 3417 variants, including $188 \mathrm{P}$ and $160 \mathrm{LP}$, for assessing genetic risk of secondary neoplasms in adulthood; and (3) the Genomes for Kids clinical research study of pediatric cancer patients (https://clinicaltrials.gov/ct2/show/ NCT02530658), which is ongoing. The expert-curated decisions for the first two published studies were also reapplied to incoming variant classification requests.

We have also engaged the CReATe Consortium to expand PeCanPIE's application for noncancer studies. Variants in 522 ALS patients from CReATe are being curated for upload to PeCanPIE. A future direction by this working group is the inclusion of tracks displaying intrinsically disordered regions (IDRs), a hallmark of known ALS pathogenic genes, which will allow for further prioritization of novel variants.

In addition to large-scale genetic variation analysis, PeCanPIE also supports individual users who may have a small number of patient samples. Currently there are 228 registered individual users involved in a variety of human genetic studies (e.g., Mendelian diseases, adult and pediatric cancers) from $>21$ countries, who have collectively submitted $>1100$ jobs for analysis.

\section{Discussion}

Although PeCanPIE's features partially overlap those of other available tools, we know of no other variant analysis system offering end-to-end processing with the same level of functionality. InterVar (Li and Wang 2017) provides some similar features,

\section{Genome Research}

www.genome.org 

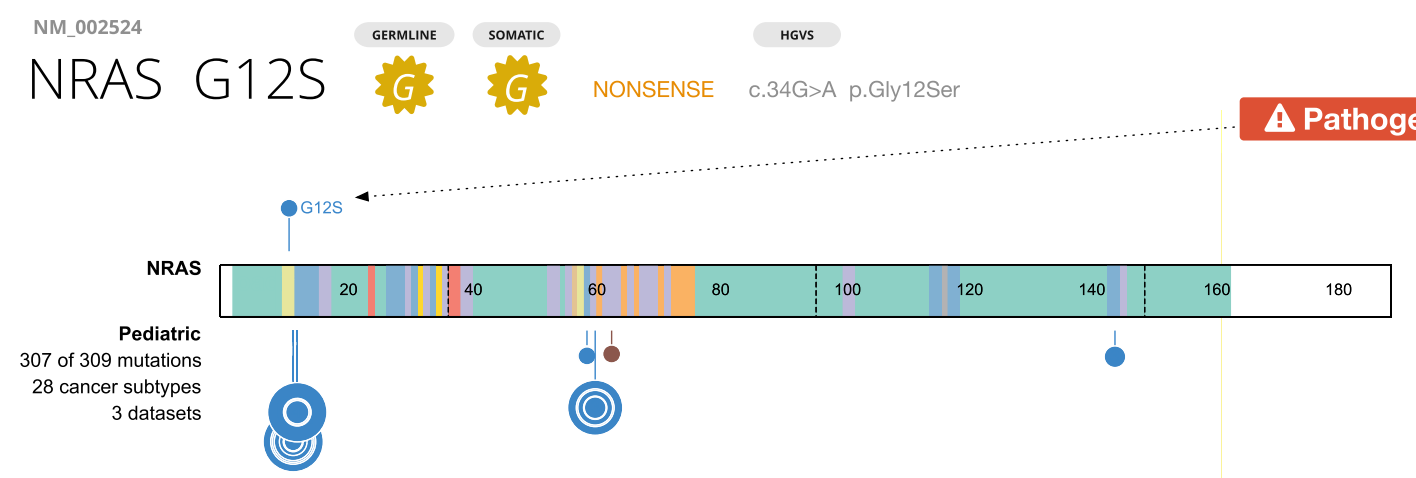

\begin{tabular}{ll}
\hline FR_N_K_Ras_like & Ras GTPase family containing H-Ras,N-Ras and K-Ras4A/4B \\
\hline Category & NONSENSE MISSENSE PROTEININS SILENT PROTEIND \& Reference \\
\hline PM1 - Moderate 4 & $\begin{array}{l}\text { Same amino acid change as a previously established pathogenic } \\
\text { variant regardless of nucleotide change } \\
\text { clinvar }\end{array}$ \\
\hline PM1 - Moderate 4 & $\begin{array}{l}\text { Located in a mutational hotspot and/or critical and well- } \\
\text { established functional domain (e.g., active site of an enzyme) } \\
\text { without benign variation } \\
\text { cosmic_hotspot }\end{array}$ \\
\hline PM2 - Moderate 4 & $\begin{array}{l}\text { Absent from controls (or at extremely low frequency if recessive) } \\
\text { in Exome Sequencing Project, 1000 Genomes Project, or Exome } \\
\text { Aggregation Consortium } \\
\text { exac_ex_tcga, 0.0001 }\end{array}$ \\
\hline
\end{tabular}

Figure 5. ACMG classification of NRAS G12S. (Top) ProteinPaint display of somatic NRAS variants across 28 cancer subtypes, showing hotspot at G12. Arrow indicates position of germline G12S variant. (Bottom) Detail of PeCanPIE ACMG classification interface for G12S variant. Automated classification detected a pathogenic ClinVar variant at the same amino acid position as well as a hotspot in COSMIC data.

however its web-based implementation can analyze only a single variant at a time and only SNVs; it cannot handle indels, MNVs, or complex substitutions, nor can it batch-process variants. The offline version is more capable; however, it relies on the semicommercial ANNOVAR software (Wang et al. 2010), which requires paid licensing even for nonprofit organizations. CIViC (Griffith et al. 2017) offers a detailed curation model, but for predisposing variants it simply directs users to manually follow the ACMG classification guidelines. The ClinGen Pathogenicity Calculator (Patel et al. 2017) offers a comprehensive ACMG classification interface, but only works with a single variant at a time, lacks automation, and cannot batch-process variants. On the other end of the spectrum, CRAVAT (Masica et al. 2017) can batch-process collections of variants, but pathogenicity is ranked based on machine learning-based VEST and CHASM scores (Carter et al. 2009, 2013); in contrast, PeCanPIE provides more granular annotations and discrete ACMG-recommended evidence tags, which allow analysts to see and weigh these individual contributions to overall variant pathogenicity.

PeCanPIE also offers additional capabilities. Novel features include (1) tight integration of variant classification with the rich resource of somatic mutation data in pediatric cancer, which can be explored online via the embedded ProteinPaint view; (2) enhancement to splice variant annotation (Methods); (3) to aid analysts with literature review, PubMed references are extracted from ClinVar, VEP, COSMIC, and locus-specific databases and hyperlinked; (4) collaborative features allow users to invite others to share results or work together on classifying sets of variants; and (5) cloud-based implementation of PeCanPIE, which obviates the need for complex software installation and command-line workflows. This design also allows back-end analysis pipelines to be invoked independently from PeCanPIE for users who prefer direct or programmatic access over a graphical interface.

A limitation of the existing method is that precomputed damage-prediction algorithm scores are taken from the dbNSFP database, which only contains data for nonsilent SNVs. Although these annotations are unavailable for indels, because protein class annotations are taken into account by the scoring algorithm, highimpact events such as truncating variations will still be highly ranked. For variant population frequency filtering, we are currently using the non-TCGA subset of ExAC instead of gnomAD (Lek et al. 2016) because the gnomAD database contains TCGA samples; we plan to migrate to gnomAD once a TCGA-subtracted version becomes publicly available. Inaccuracy in public databases, such as misannotation of a rare germline variant as a somatic hotspot mutation, may lead to an over-rated gold medal assigned by MedalCeremony, which will require literature review for final pathogenicity classification.

In conclusion, the PeCanPIE platform significantly accelerates the variant classification process by automating many prerequisite steps, helping to prioritize potentially pathogenic variants in massively parallel sequencing data, and providing a robust platform for investigating variant pathogenicity in disease-related genes. Although PeCanPIE was developed and tested with 

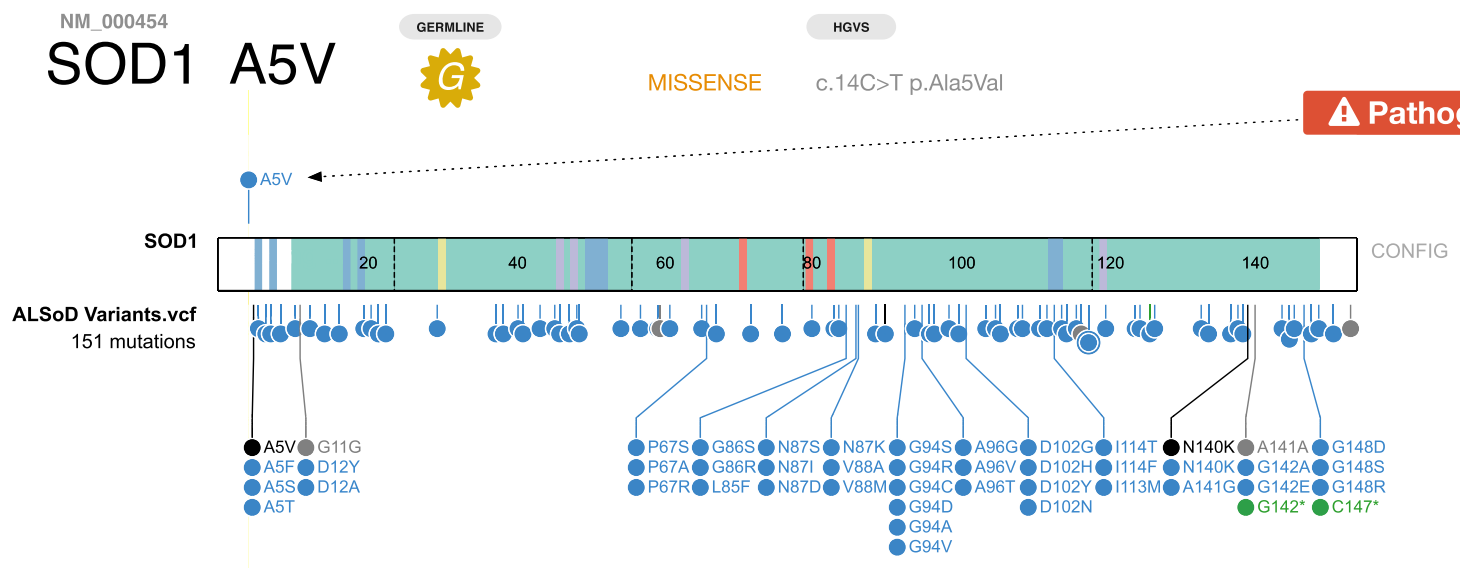

Sod_Cu. Copper/zinc superoxide dismutase (SODC)

\begin{tabular}{ll}
\hline FRAMESHIFT NONSENSE MISSENSE PROTEININS SILENT PROTEINDE & Evidence \& Reference \\
\hline Category & $\begin{array}{l}\text { PVS1 null variant (nonsense, frameshift, canonical } \pm 1 \text { or } 2 \text { splice } \\
\text { sites, initiation codon, single or multiexon deletion) in a gene where } \\
\text { PVS1 - Very Strong is a known mechanism of disease. }\end{array}$ \\
\hline PS1 - Strong 4 & $\begin{array}{l}\text { Same amino acid change as a previously established pathogenic } \\
\text { variant regardless of nucleotide change } \\
\text { Clinvar }\end{array}$ \\
\hline PS3 - Strong & $\begin{array}{l}\text { Well-established in vitro or in vivo functional studies supportive of a } \\
\text { damaging effect on the gene or gene product } \\
\text { PMID: 29748379; PMID: 29666246; }\end{array}$ \\
\hline PS4 - Strong & $\begin{array}{l}\text { The prevalence of the variant in affected individuals is significantly } \\
\text { increased compared with the prevalence in controls }\end{array}$ \\
\hline PM2 - Moderate 4 & $\begin{array}{l}\text { Absent from controls (or at extremely low frequency if recessive) in } \\
\text { Exome Sequencing Project, 1000 Genomes Project, or Exome } \\
\text { Aggregation Consortium } \\
\text { exac_ex_tcga, 0.0001 }\end{array}$ \\
\hline
\end{tabular}

Figure 6. ACMG classification of SOD1 Ala5Val. (Top) ProteinPaint display of somatic SOD1 variants; arrow indicates position of Ala5Val. (Bottom) Detail of PeCanPIE ACMG classification interface for this variant.

pediatric cancer susceptibility as the initial focus, its use is now being expanded to other pediatric and adult diseases. Users are now able to specify custom gene lists to analyze appropriately to their diseases of interest, enabling disease-specific variant curation, and facilitating gene discovery. Its unique collaborative features allow scientists with different expertise to participate in the variant classification process, as demonstrated in the use of TP53 mutagenesis data generated from yeast and mammalian model organisms (Fig. 3D). PeCanPIE provides both clinical and basic science researchers alike an intuitive platform for evaluating alleles of interest in the context of existing data.

\section{Methods}

\section{Disease-related gene lists}

Gene lists are provided for cancer- and noncancer-related diseases (Supplemental Table S1). The cancer gene list was compiled from public resources and cancer genetic studies including (1) studies of germline mutations in predisposition genes in cancer patients (Zhang et al. 2015; Huang et al. 2018; Wang et al. 2018); (2) cancer predisposition genes compiled by Rahman (Rahman 2014); (3) the
Cancer Gene Census (Futreal et al. 2004); and (4) driver genes identified in pediatric and adult pan-cancer studies (Gröbner et al. 2018; Ma et al. 2018). Publications were reviewed to confirm the presence of either loss-of-function or gain-of-function mutations in cancer driver genes, excluding those previously identified as having elevated mutation rates (e.g., LRP1B) (Lawrence et al. 2013) and those reported only as fusion partners. Other disease-related genes include cardiovascular, nonmalignant hematological, immunodeficiency, and amyotrophic lateral sclerosis (ALS)-related genes (Abel et al. 2013; Taylor et al. 2016) and genes from ACMG and Ambry Genetics incidental finding gene lists (Kalia et al. 2017). Filtering the input variants to disease-related genes helps focus on areas with relevant research interest and reduce the downstream processing burden, which is especially helpful for WGS data which may contain 4-5 million variants per sample. Users may choose to focus on one or more of these predefined disease categories for expert review or provide their own gene lists for custom analysis.

\section{Gene annotation and splice calling enhancement}

Gene annotation is performed using the VEP pipeline (McLaren et al. 2016), which provides information on a variant basis for

\section{Genome Research}

www.genome.org 
the affected gene and transcript, functional class (e.g., silent, missense, and nonsense), and effect on protein coding. We enhanced splice variant annotation by reclassifying silent or missense variants at exon boundaries, which may impact splicing (e.g., in TP53, NM_000546.5:c.375G >A, NP_000537.3:p.Thr125= and NM_000546.5:c.672G >A, NP_000537.3:p.Glu224=) (Supek et al. 2014; Soussi et al. 2017). Although not all of these variants will ultimately prove to be splice-related, these adjustments ensure additional scrutiny during expert review. A subsequent filtering step retains only variants in coding and splice-related regions. Silent variants are also kept because in rare cases they may cause aberrant splicing and thus be pathogenic. For example, ClinVar (Landrum et al. 2018) ID 90407 is a "silent" variant in the colon cancer predisposition gene MLH1 (NM_000249.3:c.882C > T, NP_000240.1: p.Leu294=) that has been determined by an expert panel to be a pathogenic splice variant (Auclair et al. 2006). We refer to this enhanced pipeline as VEP+, which may also be run independently on St. Jude Cloud.

\section{Somatic medals}

Although the PeCanPIE platform is intended to support only classification of germline variants, a basic somatic medal call is also provided to assist manual curation by considering the variant in a somatically acquired context. The somatic medal is primarily based on direct matches to somatic mutation hotspots or loss-offunction mutations in tumor suppressor genes annotated in the two somatic databases incorporated in this study, that is, PCGP and COSMIC. The separate logic can result in different medals assigned to somatic and germline. For example, the NOTCH1 R1350L variant in Figure 3 receives a bronze somatic medal and a germline silver medal; the more significant germline medal is attributable to a match in the ClinVar database, a resource that is not considered by the somatic classifier.

\section{St. Jude Cloud}

Although PeCanPIE was designed as a web portal to maximize ease of use for non-bioinformaticians, two component pipelines are also publicly accessible. On its back-end, St. Jude Cloud (https ://stjude.cloud) uses DNAnexus (https://www.dnanexus.com/), a platform in which user-created software pipelines can be installed and run on cloud computing instances. A DNAnexus account is required to use PeCanPIE for secure storage and to send notifications when submitted jobs are complete. Once a pipeline has been installed on DNAnexus, it is straightforward for nonexpert users to run it, either from a standardized web interface or a commandline client. We have installed two pipelines used by PeCanPIE on DNAnexus: VEP+ for variant annotation (app-stjude_vep_plus) and MedalCeremony for automated classification (app-stjude_medal_ceremony). These pipelines provide a wide variety of annotations which are then displayed by the PeCanPIE portal; bioinformaticians or others who would prefer direct access to the annotations can access them via the pipelines in a simple tab-delimited text format. The availability of these component pipelines on the cloud provides users and institutions straightforward, scalable access to the software, and our centralized maintenance allows all users to immediately benefit from updates and new features as they become available. An example workflow using the DNAnexus command-line client can be found in Supplemental Methods. For users who prefer not to use command-line tools, DNAnexus also provides a graphical interface for configuring and running the cloud pipelines. PeCanPIE is free for noncommercial use.

\section{Nomenclature standardization}

We have observed that various variant databases that form the foundation of annotations for PeCanPIE vary in the structure and quality of variant specification. For example, databases may provide only protein-level annotations, only genomic annotations, or both. Likewise, there are many variations on the Human Genome Variation Society (HGVS)-like protein annotation nomenclature in circulation. The PeCanPIE code attempts to be flexible in parsing, standardizing, and formatting where possible; for example, protein annotations may use either three-character or one-character protein codes (e.g., "Ser" or "S"), and a number of variations on stop codon formatting have been observed ("Ter," "Term," “*," "X," and "Stop"). In some cases, partial information such as codon numbers were extracted from an otherwise incomplete annotation. Some databases also provide variations on the five-tier ACMG pathogenicity calls which PeCanPIE attempts to standardize into B/LB/VUS/LP/P for easier comparison. We believe these standardizations further improve the reliability of annotations and utility of information provided by the PeCanPIE platform.

\section{Example data}

The ALL variants in Figure 1B were called from St. Jude sample SJNORM015857_G1 (Supplemental Methods) and uploaded to PeCanPIE. The Genome in a Bottle VCF used for Figure 1C is available from ftp://ftp-trace.ncbi.nlm.nih.gov/giab/ftp/release/ NA12878_HG001/NISTv3.3.2/GRCh37/HG001_GRCh37_GIAB_ highconf_CG-IllFB-IIlGATKHC-Ion-10X-SOLID_CHROM1-X_v.3. 3.2_highconf_PGandRTGphasetransfer.vcf.gz. This bgzip-compressed VCF file may be used directly with PeCanPIE.

\section{Software availability}

PeCanPIE is available at https://platform.stjude.cloud/tools/ pecan_pie and is one component of St. Jude Cloud (https ://stjude.cloud/). Source code for the VEP+ and MedalCeremony pipelines and all scripts generated in this study are available as Supplemental Code and from https://github.com/mnedmonson/ SJCRH/.

\section{Acknowledgments}

This project was supported by the American Lebanese Syrian Associated Charities (ALSAC) of St. Jude Children's Research Hospital, by a Cancer Center Support (Core) grant (CA21765) and a grant to J.Z. (CA21635) from the National Cancer Institute. The CReATe Consortium (U54NS092091) is part of Rare Diseases Clinical Research Network (RDCRN), an initiative of the Office of Rare Diseases Research (ORDR), NCATS. This consortium is funded through collaboration between NCATS and the NINDS. We thank Dr. Yiyang Wu for discussions of in silico algorithms.

Author contributions: Analysis pipeline design and development (M.N.E.), web software design and development (A.N.P., X.Z., and J.B.B.), cloud pipeline development (M.N.E. and C.L.M.), tool development (M.N.E., S.V.R., and M.C.R.), genomic data analysis (E.R., D.J.H., Y.L., C.A.K., J.Z., S.N., Z.W., L.L.R., A.N.P., T.S., J.P.T., M.B., and M.N.E.), manuscript text (M.N.E., J.Z., E.R., and C.A.K.), figure preparation (A.N.P., M.N.E., and J.Z.), database support (M.R.W.), and project direction and supervision (J.Z., J.R.D., and K.E.N.). 


\section{References}

The 1000 Genomes Project Consortium. 2015. A global reference for human genetic variation. Nature 526: 68-74. doi:10.1038/nature15393

Abel O, Shatunov A, Jones AR, Andersen PM, Powell JF, Al-Chalabi A. 2013. Development of a smartphone app for a genetics website: the amyotrophic lateral sclerosis online genetics database (ALSoD). JMIR Mhealth Uhealth 1: e18. doi:10.2196/mhealth.2706

Amberger JS, Bocchini CA, Schiettecatte F, Scott AF, Hamosh A. 2015 OMIM.org: Online Mendelian Inheritance in Man $\left(\mathrm{OMIM}^{\circledR}\right)$, an online catalog of human genes and genetic disorders. Nucleic Acids Res 43: D789-D798. doi:10.1093/nar/gku1205

Auclair J, Busine MP, Navarro C, Ruano E, Montmain G, Desseigne F, Saurin JC, Lasset C, Bonadona V, Giraud S, et al. 2006. Systematic mRNA analysis for the effect of MLH1 and MSH2 missense and silent mutations on aberrant splicing. Hum Mutat 27: 145-154. doi:10.1002/humu.20280

Béroud C, Letovsky SI, Braastad CD, Caputo SM, Beaudoux O, Bignon YJ Bressac-De Paillerets B, Bronner M, Buell CM, Collod-Béroud G, et al. 2016. BRCA share: a collection of clinical BRCA gene variants. Hum Mutat 37: 1318-1328. doi:10.1002/humu.23113

Bouaoun L, Sonkin D, Ardin M, Hollstein M, Byrnes G, Zavadil J, Olivier M. 2016. TP53 variations in human cancers: new lessons from the IARC TP53 database and genomics data. Hum Mutat 37: 865-876. doi:10 $.1002 /$ humu. 23035

The Cancer Genome Atlas Research Network. 2008. Comprehensive genomic characterization defines human glioblastoma genes and core pathways. Nature 455: 1061-1068. doi:10.1038/nature07385

Carter H, Chen S, Isik L, Tyekucheva S, Velculescu VE, Kinzler KW, Vogelstein B, Karchin R. 2009. Cancer-specific high-throughput annotation of somatic mutations: computational prediction of driver missense mutations. Cancer Res 69: 6660-6667. doi:10.1158/0008-5472 CAN-09-1133

Carter H, Douville C, Stenson PD, Cooper DN, Karchin R. 2013. Identifying Mendelian disease genes with the variant effect scoring tool. BMC Genomics 14: S3. doi:10.1186/1471-2164-14-S3-S3

Chakravarty D, Gao J, Phillips SM, Kundra R, Zhang H, Wang J, Rudolph JE, Yaeger R, Soumerai T, Nissan MH, et al. 2017. OncoKB: a precision oncology knowledge base. JCO Precis Oncol 1. doi:10.1200/PO.17.00011

Churchman ML, Qian M, te Kronnie G, Zhang R, Yang W, Zhang H, Lana T, Tedrick P, Baskin R, Verbist K, et al. 2018. Germline genetic IKZF1 variation and predisposition to childhood acute lymphoblastic leukemia. Cancer Cell 33: 937-948.e8. doi:10.1016/j.ccell.2018.03.021

Cingolani P, Platts A, Wang LL, Coon M, Nguyen T, Wang L, Land SJ, Lu X, Ruden DM. 2012. A program for annotating and predicting the effects of single nucleotide polymorphisms, SnpEff. Fly (Austin) 6: 80-92. doi:10 $.4161 /$ fly. 19695

Cudkowicz ME, McKenna-Yasek D, Sapp PE, Chin W, Geller B, Hayden DL, Schoenfeld DA, Hosler BA, Horvitz HR, Brown RH. 1997. Epidemiology of mutations in superoxide dismutase in amyotrophic lateral sclerosis. Ann Neurol 41: 210-221. doi:10.1002/ana.410410212

Danecek P, Auton A, Abecasis G, Albers CA, Banks E, DePristo MA, Handsaker RE, Lunter G, Marth GT, Sherry ST, et al. 2011. The variant call format and VCFtools. Bioinformatics 27: 2156-2158. doi:10.1093/ bioinformatics/btr330

Downing JR, Wilson RK, Zhang J, Mardis ER, Pui CH, Ding L, Ley TJ, Evans WE. 2012. The pediatric cancer genome project. Nat Genet 44: 619-622. doi:10.1038/ng.2287

Fokkema IF, Taschner PE, Schaafsma GC, Celli J, Laros JF, den Dunnen JT. 2011. LOVD v.2.0: the next generation in gene variant databases. Hum Mutat 32: 557-563. doi:10.1002/humu.21438

Forbes SA, Bhamra G, Bamford S, Dawson E, Kok C, Clements J, Menzies A Teague JW, Futreal PA, Stratton MR. 2008. The catalogue of somatic mutations in cancer (COSMIC). Curr Protoc Hum Genet 57: 10.11.110.11.26. doi:10.1002/0471142905.hg1011s57

Futreal PA, Coin L, Marshall M, Down T, Hubbard T, Wooster R, Rahman N, Stratton MR. 2004. A census of human cancer genes. Nat Rev Cancer 4: 177-183. doi:10.1038/nrc1299

Ghosh R, Oak N, Plon SE. 2017. Evaluation of in silico algorithms for use with ACMG/AMP clinical variant interpretation guidelines. Genome Biol 18: 225. doi:10.1186/s13059-017-1353-5

Giacomelli AO, Yang X, Lintner RE, McFarland JM, Duby M, Kim J, Howard TP, Takeda DY, Ly SH, Kim E, et al. 2018. Mutational processes shape the landscape of TP53 mutations in human cancer. Nat Genet 50: 13811387. doi:10.1038/s41588-018-0204-y

Griffith M, Spies NC, Krysiak K, McMichael JF, Coffman AC, Danos AM, Ainscough BJ, Ramirez CA, Rieke DT, Kujan L, et al. 2017. CIViC is a community knowledgebase for expert crowdsourcing the clinical interpretation of variants in cancer. Nat Genet 49: 170-174. doi:10.1038/ng .3774

Gröbner SN, Worst BC, Weischenfeldt J, Buchhalter I, Kleinheinz K, Rudneva VA, Johann PD, Balasubramanian GP, Segura-Wang M,
Brabetz S, et al. 2018. The landscape of genomic alterations across childhood cancers. Nature 555: 321-327. doi:10.1038/nature 25480

Hinrichs AS, Karolchik D, Baertsch R, Barber GP, Bejerano G, Clawson H, Diekhans M, Furey TS, Harte RA, Hsu F, et al. 2006. The UCSC Genome Browser Database: update 2006. Nucleic Acids Res 34: D590D598. doi:10.1093/nar/gkj144

Huang K, Mashl RJ, Wu Y, Ritter DI, Wang J, Oh C, Paczkowska M, Reynolds S, Wyczalkowski MA, Oak N, et al. 2018. Pathogenic germline variants in 10,389 adult cancers. Cell 173: 355-370.e14. doi:10.1016/j.cell .2018.03.039

Ioannidis NM, Rothstein JH, Pejaver V, Middha S, McDonnell SK, Baheti S Musolf A, Li Q, Holzinger E, Karyadi D, et al. 2016. REVEL: an ensemble method for predicting the pathogenicity of rare missense variants. Am J Hum Genet 99: 877-885. doi:10.1016/j.ajhg.2016.08.016

Kalia SS, Adelman K, Bale SJ, Chung WK, Eng C, Evans JP, Herman GE, Hufnagel SB, Klein TE, Korf BR, et al. 2017. Recommendations for reporting of secondary findings in clinical exome and genome sequencing, 2016 update (ACMG SF v2.0): a policy statement of the American College of Medical Genetics and Genomics. Genet Med 19: 249-255. doi:10.1038/gim.2016.190

Kanchi KL, Johnson KJ, Lu C, McLellan MD, Leiserson MD, Wendl MC, Zhang Q, Koboldt DC, Xie M, Kandoth C, et al. 2014. Integrated analysis of germline and somatic variants in ovarian cancer. Nat Commun 5: 3156. doi:10.1038/ncomms4156

Kotler E, Shani O, Goldfeld G, Lotan-Pompan M, Tarcic O, Gershoni A, Hopf TA, Marks DS, Oren M, Segal E. 2018. A systematic p53 mutation library links differential functional impact to cancer mutation pattern and evolutionary conservation. Mol Cell 71: 178-190.e8. doi:10.1016/j.molcel .2018.06.012

Landrum MJ, Lee JM, Benson M, Brown GR, Chao C, Chitipiralla S, Gu B, Hart J, Hoffman D, Jang W, et al. 2018. ClinVar: improving access to variant interpretations and supporting evidence. Nucleic Acids Res 46: D1062-D1067. doi:10.1093/nar/gkx1153

Lawrence MS, Stojanov P, Polak P, Kryukov G V, Cibulskis K, Sivachenko A Carter SL, Stewart C, Mermel CH, Roberts SA, et al. 2013. Mutationa heterogeneity in cancer and the search for new cancer-associated genes. Nature 499: 214-218. doi:10.1038/nature12213

Lek M, Karczewski KJ, Minikel E V, Samocha KE, Banks E, Fennell T, O'Donnell-Luria AH, Ware JS, Hill AJ, Cummings BB, et al. 2016 Analysis of protein-coding genetic variation in 60,706 humans. Nature 536: 285-291. doi:10.1038/nature 19057

Li Q, Wang K. 2017. InterVar: clinical interpretation of genetic variants by the 2015 ACMG-AMP guidelines. Am J Hum Genet 100: 267-280. doi:10 .1016/j.ajhg.2017.01.004

Liu X, Jian X, Boerwinkle E. 2013. dbNSFP v2.0: a database of human nonsynonymous SNVs and their functional predictions and annotations. Hum Mutat 34: E2393-E2402. doi:10.1002/humu.22376

Lohmann DR, Gallie BL. 1993. Retinoblastoma. In GeneReviews [Internet] (ed. Adam MP, et al.). University of Washington, Seattle. https://www ncbi.nlm.nih.gov/pubmed/20301625 [accessed May 21, 2018].

Ma X, Liu Y, Liu Y, Alexandrov LB, Edmonson MN, Gawad C, Zhou X, Li Y, Rusch MC, Easton J, et al. 2018. Pan-cancer genome and transcriptome analyses of 1,699 paediatric leukaemias and solid tumours. Nature 555: 371-376. doi:10.1038/nature25795

Margraf RL, Crockett DK, Krautscheid PMF, Seamons R, Calderon FRO, Wittwer CT, Mao R. 2009. Multiple endocrine neoplasia type 2 RET protooncogene database: repository of MEN2-associated RET sequence variation and reference for genotype/phenotype correlations. Hum Mutat 30: 548-556. doi:10.1002/humu.20928

Masica DL, Douville C, Tokheim C, Bhattacharya R, Kim R, Moad K, Ryan MC, Karchin R. 2017. CRAVAT 4: cancer-related analysis of variants toolkit. Cancer Res 77: e35-e38. doi:10.1158/0008-5472.CAN-17-0338

McLaren W, Gil L, Hunt SE, Riat HS, Ritchie GR, Thormann A, Flicek P, Cunningham F. 2016. The Ensembl Variant Effect Predictor. Genome Biol 17: 122. doi:10.1186/s13059-016-0974-4

Moriyama T, Metzger ML, Wu G, Nishii R, Qian M, Devidas M, Yang W, Cheng C, Cao X, Quinn E, et al. 2015. Germline genetic variation in ETV6 and risk of childhood acute lymphoblastic leukaemia: a systematic genetic study. Lancet Oncol 16: 1659-1666. doi:10.1016/S1470-2045 (15)00369-1

Ng SB, Turner EH, Robertson PD, Flygare SD, Bigham AW, Lee C, Shaffer T, Wong M, Bhattacharjee A, Eichler EE, et al. 2009. Targeted capture and massively parallel sequencing of 12 human exomes. Nature 461: 272276. doi:10.1038/nature08250

Patel RY, Shah N, Jackson AR, Ghosh R, Pawliczek P, Paithankar S, Baker A, Riehle K, Chen H, Milosavljevic S, et al. 2017. ClinGen Pathogenicity Calculator: a configurable system for assessing pathogenicity of genetic variants. Genome Med 9: 3. doi:10.1186/s13073-016-0391-z

Podlevsky JD, Bley CJ, Omana R V, Qi X, Chen JJ. 2007. The telomerase database. Nucleic Acids Res 36: D339-D343. doi:10.1093/nar/gkm700 
Rahman N. 2014. Realizing the promise of cancer predisposition genes. Nature 505: 302-308. doi:10.1038/nature 12981

Richards S, Aziz N, Bale S, Bick D, Das S, Gastier-Foster J, Grody WW, Hegde M, Lyon E, Spector E, et al. 2015. Standards and guidelines for the interpretation of sequence variants: a joint consensus recommendation of the American College of Medical Genetics and Genomics and the Association for Molecular Pathology. Genet Med 17: 405-423. doi:10 .1038 /gim.2015.30

Rosen DR, Bowling AC, Patterson D, Usdin TB, Sapp P, Mezey E, McKennaYasek D, O'Regan J, Rahmani Z, Ferrante RJ. 1994. A frequent ala 4 to val superoxide dismutase- 1 mutation is associated with a rapidly progressive familial amyotrophic lateral sclerosis. Hum Mol Genet 3: 981-987. doi: $10.1093 / \mathrm{hmg} / 3.6 .981$

Schubbert S, Shannon K, Bollag G. 2007. Hyperactive Ras in developmental disorders and cancer. Nat Rev Cancer 7: 295-308. doi:10.1038/nrc2109

Shah S, Schrader KA, Waanders E, Timms AE, Vijai J, Miething C, Wechsler J, Yang J, Hayes J, Klein RJ, et al. 2013. A recurrent germline PAX5 mutation confers susceptibility to pre-B cell acute lymphoblastic leukemia. Nat Genet 45: 1226-1231. doi:10.1038/ng.2754

Soussi T, Asselain B, Hamroun D, Kato S, Ishioka C, Claustres M, Béroud C. 2006. Meta-analysis of the p53 mutation database for mutant p53 biological activity reveals a methodologic bias in mutation detection. Clin Cancer Res 12: 62-69. doi:10.1158/1078-0432.CCR-05-0413

Soussi T, Taschner PE, Samuels Y. 2017. Synonymous somatic variants in human cancer are not infamous: a plea for full disclosure in databases and publications. Hum Mutat 38: 339-342. doi:10.1002/humu.23163

Supek F, Miñana B, Valcárcel J, Gabaldón T, Lehner B. 2014. Synonymous mutations frequently act as driver mutations in human cancers. Cell 156: $1324-1335$. doi:10.1016/j.cell.2014.01.051

Szabo C, Masiello A, Ryan JF, Brody LC. 2000. The breast cancer information core: database design, structure, and scope. Hum Mutat 16: 123-131. doi:10.1002/1098-1004(200008)16:2<123::AID-HUMU4>3.0.CO;2-Y

Taylor JP, Brown RH, Cleveland DW. 2016. Decoding ALS: from genes to mechanism. Nature 539: 197-206. doi:10.1038/nature20413
Valentine JS, Hart PJ. 2003. Misfolded CuZnSOD and amyotrophic lateral sclerosis. Proc Natl Acad Sci 100: 3617-3622. doi:10.1073/pnas .0730423100

Wang K, Li M, Hakonarson H. 2010. ANNOVAR: functional annotation of genetic variants from high-throughput sequencing data. Nucleic Acids Res 38: e164. doi:10.1093/nar/gkq603

Wang Z, Wilson CL, Easton J, Thrasher A, Mulder H, Liu Q, Hedges DJ, Wang S, Rusch MC, Edmonson MN, et al. 2018. Genetic risk for subsequent neoplasms among long-term survivors of childhood cancer. J Clin Oncol 36: 2078-2087. doi:10.1200/JCO.2018.77.8589

Wildeman M, van Ophuizen E, den Dunnen JT, Taschner PE. 2008. Improving sequence variant descriptions in mutation databases and literature using the Mutalyzer sequence variation nomenclature checker. Hum Mutat 29: 6-13. doi:10.1002/humu.20654

Zhang J, Walsh MF, Wu G, Edmonson MN, Gruber TA, Easton J, Hedges D, Ma X, Zhou X, Yergeau DA, et al. 2015. Germline mutations in predisposition genes in pediatric cancer. $N$ Engl J Med 373: 2336-2346. doi:10.1056/NEJMoa1508054

Zhao M, Kim P, Mitra R, Zhao J, Zhao Z. 2016. TSGene 2.0: an updated literature-based knowledgebase for tumor suppressor genes. Nucleic Acids Res 44: D1023-D1031. doi:10.1093/nar/gkv1268

Zhou X, Edmonson MN, Wilkinson MR, Patel A, Wu G, Liu Y, Li Y, Zhang Z, Rusch MC, Parker M, et al. 2015. Exploring genomic alteration in pediatric cancer using ProteinPaint. Nat Genet 48: 4-6. doi:10.1038/ng.3466

Zook JM, Chapman B, Wang J, Mittelman D, Hofmann O, Hide W, Salit M. 2014. Integrating human sequence data sets provides a resource of benchmark SNP and indel genotype calls. Nat Biotechnol 32: 246-251. doi:10.1038/nbt.2835

Received March 13, 2019; accepted in revised form July 23, 2019. 


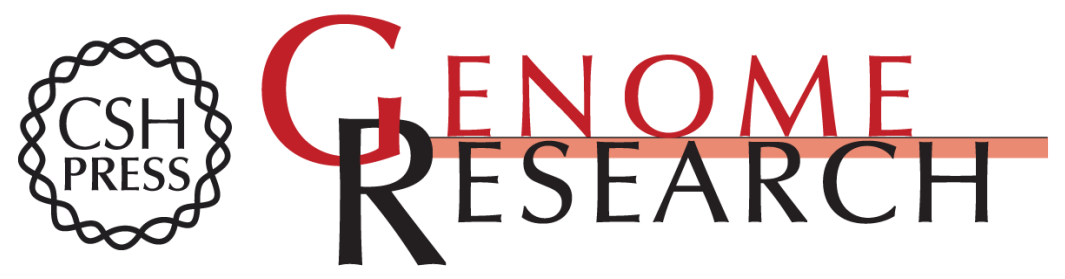

\section{Pediatric Cancer Variant Pathogenicity Information Exchange (PeCanPIE): a cloud-based platform for curating and classifying germline variants}

Michael N. Edmonson, Aman N. Patel, Dale J. Hedges, et al.

Genome Res. 2019 29: 1555-1565 originally published online August 22, 2019

Access the most recent version at doi:10.1101/gr.250357.119

Supplemental
Material http://genome.cshlp.org/content/suppl/2019/08/22/gr.250357.119.DC1

References This article cites 59 articles, 4 of which can be accessed free at:

http://genome.cshlp.org/content/29/9/1555.full.html\#ref-list-1

Open Access Freely available online through the Genome Research Open Access option.

Creative This article, published in Genome Research, is available under a Creative

Commons Commons License (Attribution 4.0 International), as described at

License http://creativecommons.org/licenses/by/4.0/.

Email Alerting Receive free email alerts when new articles cite this article - sign up in the box at the Service top right corner of the article or click here.

\section{Affordable, Accurate Sequencing.}

To subscribe to Genome Research go to:

https://genome.cshlp.org/subscriptions

(C) 2019 Edmonson et al.; Published by Cold Spring Harbor Laboratory Press 